\title{
Power Semiconductor Trends in Electric Drive Systems
}

\author{
M. Csizmadia ${ }^{1}$, M. Kuczmann ${ }^{1}$ \\ ${ }^{1}$ Széchenyi István University, Automation Department, \\ Egyetem tér 1., H-9026 Győr, Hungary \\ e-mail: csizmadia.miklos@sze.hu, kuczmann@sze.hu
}

Abstract: Nowadays lots of big brands (like Tesla, Nissan, Audi etc.) deal with electric cars and electric drive systems. The first brand deals with only electric drive systems and everyone know this name. These cars are more environmentally friendly, because those operate only with electric energy (this article does not deal with the source of electric energy). The design of electric drive systems is very difficult and complicated task: the electric, thermal and mechanical parameters are very important during the design process. The task is given: it must be designed to reach the most efficient drive system with a low cost. This article deals with the current semiconductor trends and properties, investigates the current electric car drive systems (semiconductor design perspective) and deals with the future trends.

Keywords: electric cars, power semiconductors, power electric, electric drive system, wide bandgap semiconductor

\section{Overview}

Most of electric cars drive system can be divided into the following parts:

- Battery;

- Control electronics;

- Electrical motor;

- Power electronics.

This article is deal with the power electronics, which include (Fig. 1.):

- The three-phase frequency converter, which includes the semiconductors and heat sink; 
- The gate drive systems and protection circuits;

- The current and voltage measuring systems;

- The battery management system (BMS) and the battery charger;

- The printed circuit board (PCB) design.

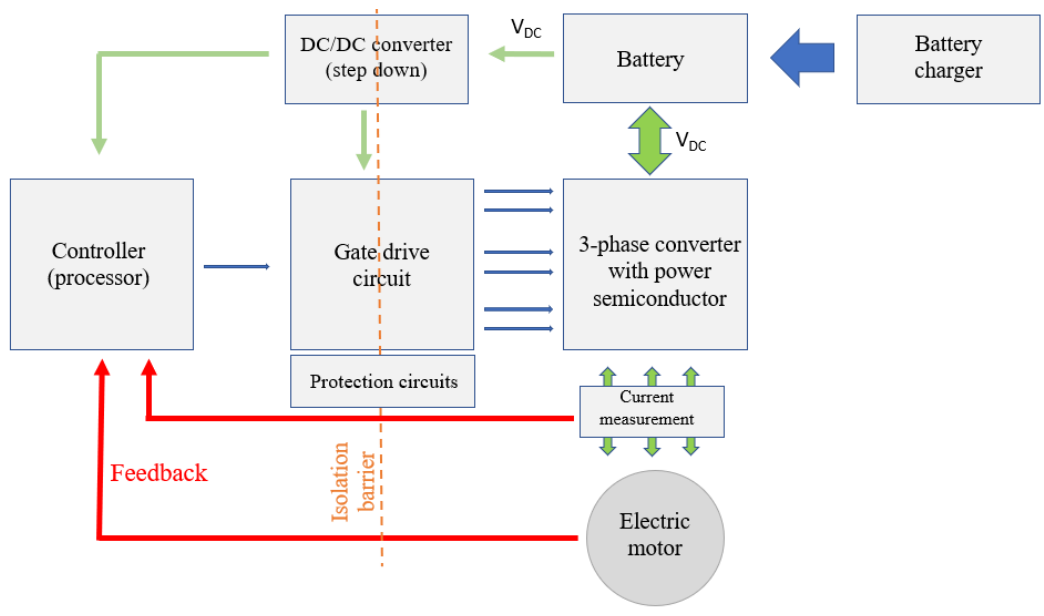

Figure 1. Block diagram of powertrain of electric cars

\section{Semiconductors in electric drive system}

Semiconductor properties extend to electrical and thermal parameters. The most important electrical parameter is the maximal values, which limit the boundary of operation:

- The breakdown voltage;

- The maximum current, which depends on the frequency and the temperature. In this case it is distinguished normal and surge current (the latest can work in short time);

- Working frequency;

- The switching parameters (capacities and charges);

- $\quad$ and their combined parameters: FBSOA (Forward Biased Safe Operating Area) and RBSOA (Reverse Bias Safe Operating Area).

Nowadays, in the high-power systems are used Insulated-Gate Bipolar Transistors (IGBTs), having high breakdown voltage and high current value, but these parameters are achieved at the cost of switching achievement. A good example is 
the Tesla Model S: the frequency converter of the car was developed with discrete IGBTs [1].

In the last decade more attention is given to the wide bandgap semiconductors such as the silicon carbide ( $\mathrm{SiC})$ and the gallium nitride $(\mathrm{GaN})$. They are called as the "next generation of the semiconductor devices". These semiconductors are the keys to the high frequency and high efficiency design systems. The high frequency switching includes the lower ripple: However, the lower ripple reduces the EMI disturbances. The high switching frequency means also higher switching losses. Therefore, the choosing of the switching frequency is an important step in the design procedure, for the high system efficiency.

The SiC-based semiconductors have lot of positive advantages: lower power loss, lower on-state resistance, greater bandwidth, which includes the higher switching frequency and the better thermal parameters. Compared to the IGBT the SiC MOSFET in the same switching frequency has lower power loss. First, in the market in Tesla Model $3 \mathrm{SiC}$-based power electronics are used with integrated gate driver [2] [3].

The common features of GaN-based transistors are the low power loss, the low on-state resistance, the very low input capacitance and the high switching frequency. Also, in case of GaN-based transistors can be found the depletion and the enhancement type. In the practice, the enhancement type is the widespread, because it is off in case of zero gate bias. A new type of GaN based semiconductor has been investigated, called the GaN E-HEMT (GaN Enhancement mode High Electron Mobility Transistor). Basically, it characterizes the same properties, as the SiCbased semiconductors, but it has more positive advantage over the SiC: very high switching frequency $(>100 \mathrm{MHz})$, low gate charge and threshold voltage, the structure does not contain "body-diode" (zero reverse recovery loss), very good gate bias properties [3] [4].

The trend shows, that the switching frequency of the system is increasing, therefore the $\mathrm{SiC}$ and the $\mathrm{GaN}$ based semiconductor will enable innovative improvements in power density, efficiency and costs in the next years.

Table 1. contains the actual (or potentially) applicable semiconductors in electric drive systems nowadays and in the future. The values in the table are based semiconductor manufacturer datasheets. Extremist examples can also be found. 
Table 1. Semiconductor properties

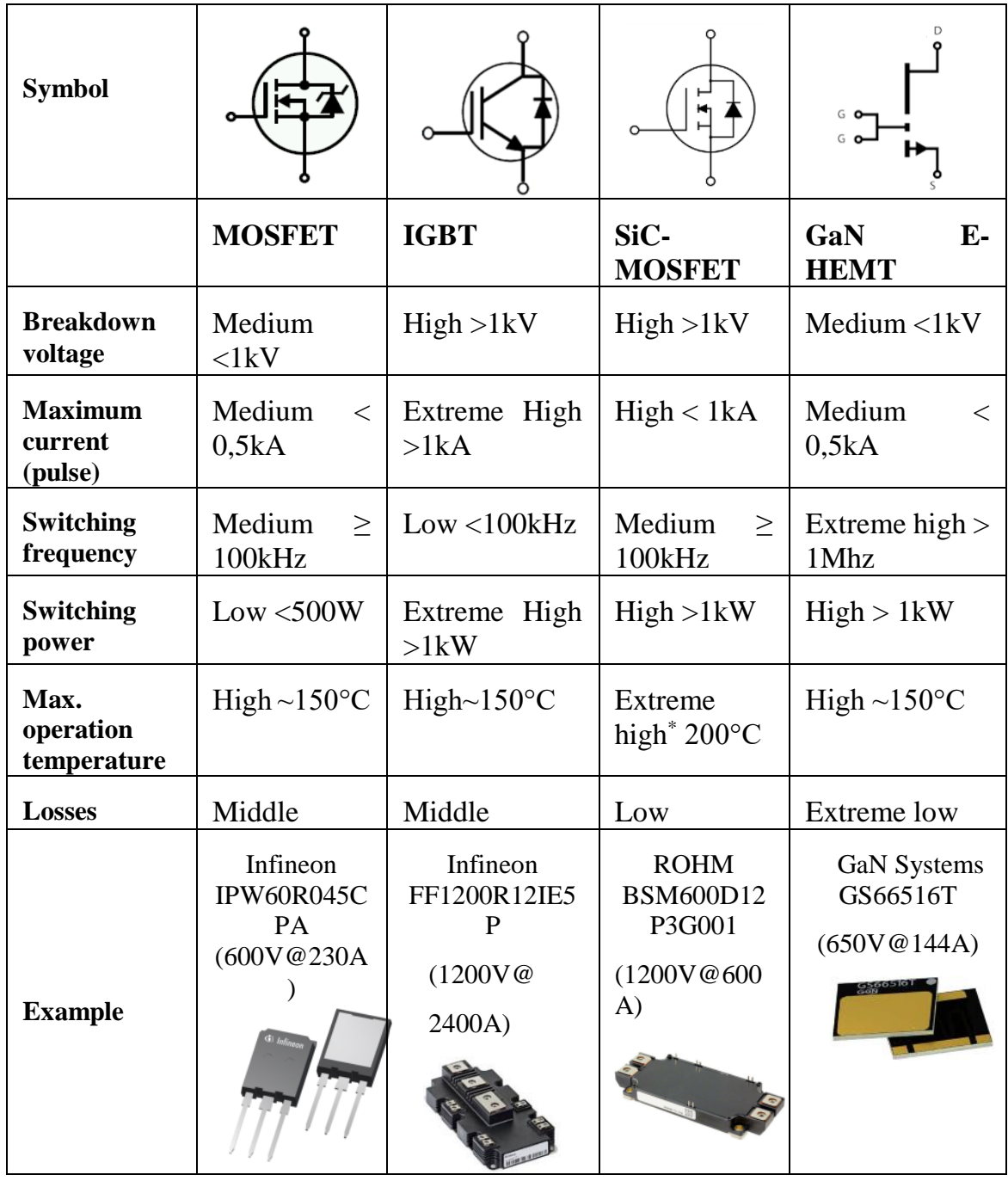

* Depend on the semiconductor casing. 


\subsection{Casing of power semiconductor}

Semiconductors design can be discrete or modular design. In the case of modular design, the semiconductors contain half bridge or three phase bridge. Sometimes the modular case is very useful, because the warming of the semiconductors is the same (if the load is the same and symmetrical). In most cases the modular design contains NTC resistors, which can be able log the thermal behavior. The other advantageous property of the modular design, of the direct liquid cooling (DLC). The high current plugs can be connected with screw and the controller plugs can be connected with soldering. In case of discrete design, the robust casing of semiconductors is recommended (e.g. TO-247): The robust case can be fixed better to the heat sink. Select of casing is depending on the design procedures: For example, in the frequency converter of Tesla Model S discrete IGBT semiconductors have been used, so this solution is not rare in the practice [1].

Another important property is the inductance of casing. At higher switching frequency the parasitic inductance has a bigger impact: the leakage inductance can cause gate ringing in the switching procedures. It can be reduced with several methods, like the distance between the gate driver and semiconductor have to be minimized. If the capacitances and parasitic inductances of the semiconductor devices are small, then the impact of these phenomena is negligible. For example, in case of TO-247 casing this parasitic inductance is about between $5 \mathrm{nH}$ and $15 \mathrm{nH}$ [6].

In case of the wide bandgap semiconductors the casing must be carefully reconsidered, because such type of semiconductor's working temperature will be higher, than the Si-based one. The requirements of the packaging are the low parasitic inductance, the mechanical robustness and the low losses. Nowadays the surface mount casing in case of high-power semiconductors is not rare. Table 1 . contains some type of casing.

\subsection{Dynamic properties of semiconductor}

The dynamic properties of the semiconductors include the switching properties: the capacitances, the charges and the inductances. These properties are defined in the datasheet, like the input, output and transfer capacitance, the gate charge and the parasitic inductance of the semiconductor.

In case of Si-based semiconductors, the input capacitance has very high value, typically a multiple thousand $\mathrm{pF}$. The more capacitance means also more gate charge, which must be moved during the switching procedures. Thus, requires the gate driver more source and sink current, which increase the complexity of the system. 
For the good comparability some 650V MOSFET with the same current rating (about $60 \mathrm{~A}$, at $25^{\circ} \mathrm{C}$ ) have been investigated. The Si-based MOSFET (STW65N65DM2AG), the SiC-based (SCT3030AL) and the GaN-based (GS66516T) are shown in Fig. 4. It has been found that the device input capacitance $\mathrm{C}_{\mathrm{iss}}=\mathrm{C}_{\mathrm{gs}}+\mathrm{C}_{\mathrm{gd}}$ of GaN-based MOSFET is the lowest, the Si-based MOSFET is slightly bigger than $\mathrm{SiC}$. Reverse transfer capacitance $\mathrm{C}_{\mathrm{rss}}=\mathrm{C}_{\mathrm{gd}}$ of GaN-HEMT is much smaller than that of $\mathrm{SiC}$ devices. Semiconductors output capacitance $\mathrm{C}_{\text {oss }}=$ $\mathrm{C}_{\mathrm{ds}}+\mathrm{C}_{\mathrm{gd}}$ values of the aforementioned devices are similar in case of $\mathrm{SiC}$ and $\mathrm{GaN}$. The output capacitance of Si-based is significantly bigger.

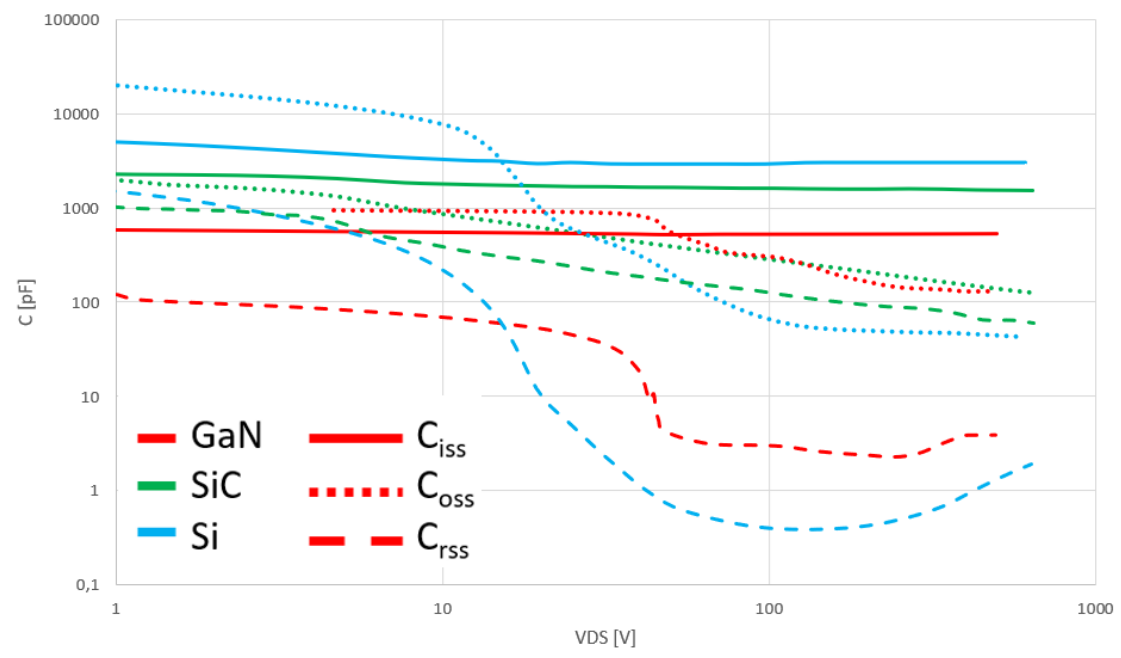

Figure 2. Comparison of Si, SiC and GaN-based semiconductor capacitance

\subsection{Body diodes}

In electric drive systems the diodes play an important role, because these conduct the current on the load during the semiconductor in off-state, or in synchronous rectifier system. The important properties of these diodes are the forward drop voltage $\left(\mathrm{V}_{\mathrm{f}}\right)$, the reverse recovery charge $\left(\mathrm{Q}_{\mathrm{rr}}\right)$ and time $\left(\mathrm{t}_{\mathrm{rr}}\right)$. To achieve high switching frequency, low reverse recovery properties are needed. In case of the Sibased semiconductors these parameters are hundreds of ns and $\mu \mathrm{C}$ in magnitude. The same parameters in case of wide bandgap semiconductors are smaller: usually less, then 100ns and the recovery charge is $\mathrm{nC}$ in magnitude [7].

In case of GaN E-HEMT the structure does not contain the "classical" body diodes. The properties of the new solution are the same with better switching 
parameters. Table 2. compares the MOSFET and the GaN E-HEMT semiconductors on and off states, focusing on the equivalent circuit [6].

Not rare the hybrid solution in high power inverters: the $\mathrm{Si}$ (body) diode are replaced by SiC Schottky diodes and are used with Si-based IGBTs. The power losses can be reduced significantly.

Table 2. Reverse conduction [6]

\begin{tabular}{|l|c|c|}
\hline Gate state & MOSFET & GaN E-HEMT \\
\hline ON & & \\
\hline OFF & & \\
& & \\
\hline
\end{tabular}

\subsection{Gate bias voltage}

Every semiconductor has a minimum voltage in the control inputs, by which the gate driver have to achieve and have to exceed, for procedures of the safe switch-on. This parameter is the well-known gate threshold voltage. The threshold voltage decreases by increasing temperature therefore the silicon material has lower threshold voltage: the gate threshold voltage has a decreasing tendency in case of new semiconductors. Therefore, the semiconductor gate voltage is a very important parameter. The output parameters of semiconductors, the gate drive losses are depending on this parameter. It is well known, that the gate driver losses are given by [8] [9]:

$$
P_{\text {loss_gate }}=Q_{g} V_{D R V} f_{\text {sw }},
$$

where

$Q_{g} \quad$ is the total gate charge (or often denoted by $\mathrm{Q}_{\mathrm{sw}}$ );

$V_{D R V}$ is the gate driver voltage;

$f_{s w} \quad$ is the switching frequency. 
The gate is fully charged and discharged in each switching period, therefore is very useful, if the value of the gate charge is small. The losses can be decreased, if the gate drive voltage is well-chosen. The basic drive method is the same as that for IGBT's and Si-MOSFET's. The turn on gate voltage is typically between $12 \mathrm{~V}-18 \mathrm{~V}$ and the turn off voltage is in the most cases negative, approximately $-3 \mathrm{~V}$ to $-8 \mathrm{~V}$. The maximum value of the gate voltage is typically $\pm 20 \mathrm{~V}$. In case of $\mathrm{GaN}$ transistors the threshold voltage is lower, therefore the gate drive voltage can be lower in the turn on procedure. The on-off gate voltage hysteresis from $-3 \mathrm{~V}$ to $+7 \mathrm{~V}$ and the maximum ratings are between $-20 \mathrm{~V}$ and $+20 \mathrm{~V}$. Table 2 . summarizes the gate bias level of the mentioned semiconductors [6].

Table 3. Gate bias level of semiconductors

\begin{tabular}{|l|l|l|l|l|}
\hline & Si-MOSFET & IGBT** & $\begin{array}{l}\text { SiC- } \\
\text { MOSFET }\end{array}$ & $\begin{array}{l}\text { GaN } \\
\text { Transistor }\end{array}$ \\
\hline $\begin{array}{l}\text { Threshold } \\
\text { voltage }\end{array}$ & $1-4 \mathrm{~V}$ & about 4V & about $3 \mathrm{~V}$ & $1-2 \mathrm{~V}$ \\
\hline $\begin{array}{l}\text { Maximum } \\
\text { rating* }\end{array}$ & $\pm 20 \mathrm{~V}$ & $\pm 20 \mathrm{~V}$ & $\pm 25 \mathrm{~V}$ & $-10 \mathrm{~V} /+7 \mathrm{~V}$ \\
\hline $\begin{array}{l}\text { Typical gate } \\
\text { bias value }\end{array}$ & $0 \mathrm{~V} / 10-12 \mathrm{~V}$ & $-8 \mathrm{~V} /+15-18 \mathrm{~V}$ & $-4 \mathrm{~V} /+15-20 \mathrm{~V}$ & 0 or $-3 \mathrm{~V} / 5-6 \mathrm{~V}$ \\
\hline
\end{tabular}

* The maximum values are different in case of different manufacturers

** The threshold voltage of high voltage devices is typically larger then for low voltage (e.g. $30 \mathrm{~V})$.

\section{Semiconductor losses}

In the switching procedures the total loss on semiconductor can be divided into two significant parts: the static losses (conducting losses) and the dynamic losses (switching losses). During the switching procedures the gate driver has a power loss, too, which can be estimated by (1).

The static losses depend on just the on-state resistance of the semiconductor and the switching current. In case of MOSFET the on-sate resistance is described by an ohmic resistance. In all other cases it can be described by the linear approximation of the U-I characteristic. The well-known equation in case of MOSFET is a follows [4] [9]:

$$
P_{\text {loss_cond }}=R_{D S(\text { on })} I_{D(R M S)}^{2}
$$


where

$\mathrm{R}_{\mathrm{DS}(\text { (on) }}$ is the semiconductor on-state resistance;

$\mathrm{I}_{\mathrm{D}(\mathrm{RMS})}$ is the root-mean-square (RMS) current through the MOSFET.

It is very important, that the static losses are independent from the frequency. The on-state resistance of the semiconductors has decreasing tendency, it is shown in Fig. 3.

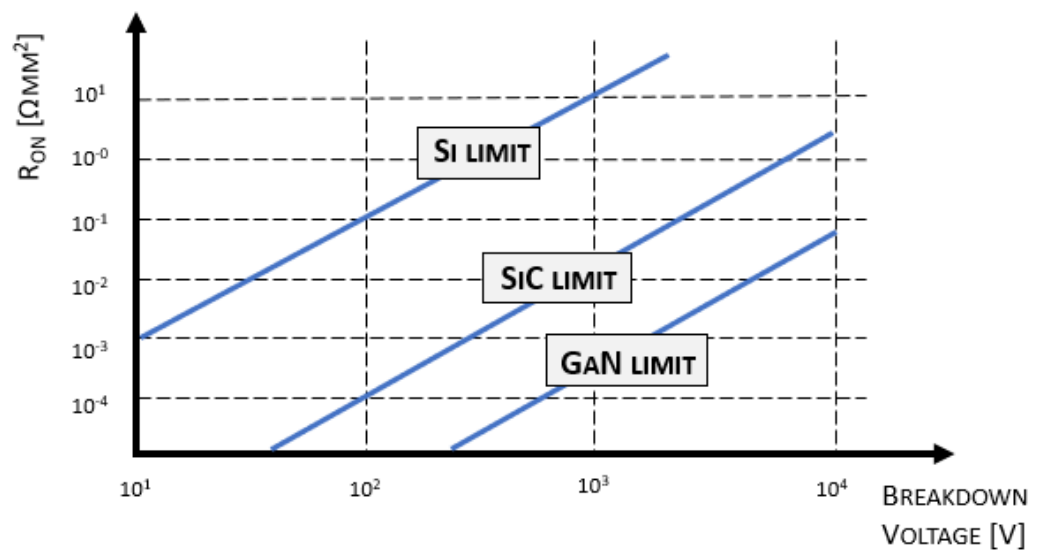

Figure 3. On-state resistance vs. blocking voltage for Si, SiC and GaN [5]

The dynamic losses are generated during the turn-on and the turn-off states, therefore the losses depending on the turn-on and turn-off energy, and the switching frequency [4] [9].

$$
P_{\text {loss_switch }}=\left(E_{\text {on }}+E_{\text {off }}\right) f_{\text {sw }} \text {. }
$$

where

$E_{\text {on }} \quad$ is the turn on energy of the semiconductor;

$E_{\text {off }} \quad$ is the turn off energy of the semiconductor;

$\mathrm{f}_{\mathrm{sw}} \quad$ is the switching frequency.

In case of diode (body diode) is distinguishable (in generally) the conduction and the switching loss (4), (5) [9]:

$$
P_{\text {loss_cond }}=V_{d} I_{f}
$$

where

$\mathrm{V}_{\mathrm{d}} \quad$ is the diode drop voltage; 
$\mathrm{I}_{\mathrm{f}} \quad$ is the current through the diode.

$$
P_{\text {loss_switch }}=\frac{1}{4} Q_{r r} V_{r} f_{\text {sw }},
$$

where

$\mathrm{Q}_{\mathrm{rr}} \quad$ is the reverse recovery charge;

$\mathrm{V}_{\mathrm{r}} \quad$ is the reverse drop voltage;

$\mathrm{f}_{\mathrm{sw}} \quad$ is the switching frequency.

\section{Parallel connection of the power semiconductors}

The high-power systems make the parallel connection of semiconductors necessary. This is possible in case of discrete or modular design, too. The aim of parallel connections is increasing of the system current or increasing the redundancy of the system. The current shares equal between the semiconductors. If the current is equally, then the warming and the losses will be equal, too. If that does not happen, then thermal difference is generated, which can increase the losses or damage the semiconductor. It is very important, that the semiconductors should have to have the same value gate of resistor.

However, the parallel connection can decrease the equivalent resistance, on the same time it means more capacitance, and hence, more charge. This will increase the switching losses at high switching frequency.

\subsection{Parallel connection of MOSFET's}

In case of MOSFET the drain-source channel can be represented by a resistor, which behaves as PTC resistor. If the temperature increases on the semiconductor, then $R_{D S}$ will be higher. Thermal differential is not formed, because the higher resistivity MOSFET conducts smaller current and forcing the higher current to the other semiconductor. This property makes the parallel connection of the MOSFET easy. In case of GaN-based semiconductor it is the same way (self-balancing) [10].

\subsection{Parallel connection of IGBT's}

In case of parallel connection of IGBT there are two important properties: the saturation voltage and the $\mathrm{U}_{\mathrm{GE}}$ and $\mathrm{I}_{\mathrm{D}}$ transfer characteristic. The latest is the same, as the MOSFET $\mathrm{R}_{\mathrm{DS}(\mathrm{on})}$ parameter. Both parameters are depending on the temperature. The thermal coefficient IGBT is a little bit more complex, as in case the MOSFET. The transfer characteristic of IGBTs contains an isothermal point: above this point the IGBT has PTC behavior (in case of higher temperature the onstate resistance is increasing), under this point the IGBT has NTC behavior. During 
the design the aim is the equal distribution of the load, therefore it must strive the PTC behavior [11].

The behavior of the semiconductor depends on the $\mathrm{U}_{\mathrm{GE}}$ voltage. If the setting of $\mathrm{U}_{\mathrm{GE}}$ voltage is right, then the semiconductor is self-regulated: if any IGBT has higher current, then the temperature on the IGBT will be higher, therefore the on-state resistance is increasing. If the aim of symmetrical operation, then each IGBTs must have the same gate resistor [11].

\section{Conclusion}

In this paper the current semiconductor trend in the electric drive system has been introduced and the opportunities of the future has been investigated. Nowadays the industry and the developers use IGBTs in high power systems, but the wide bandgap semiconductors offer new opportunities. The range, the efficiency and the switching frequency are increasing in the electric drive systems, therefore the size of the circuits and reactant parts, and their heat sink will be smaller, which mean smaller volume, size and weight. These new capabilities enable the designers to find an optimal approach to enhance the performance, reduce the losses. Fig. 4. illustrates a possible future trend.

In the future will be designed a step-down converter with GaN E-HEMT transistor and Linear-quadratic controller for the best efficiency.

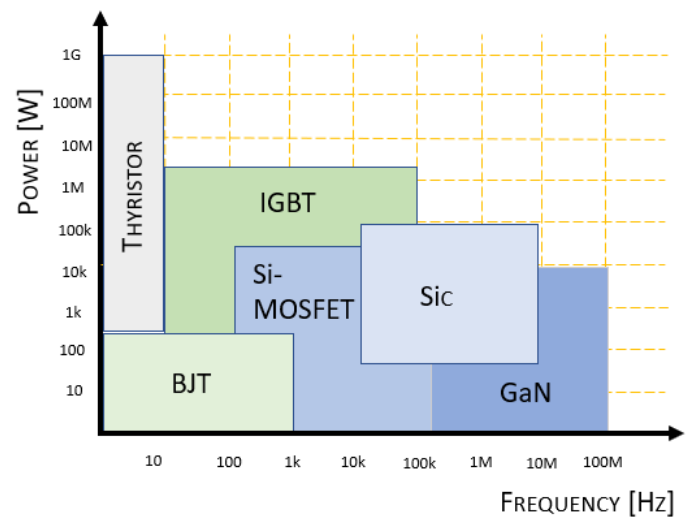

Figure 4. Possible future trend in semiconductors [12]

\section{Acknowledgement}

The publishing of this paper was supported by the project "EFOP-3.6.1-16-201600017 Internationalisation, initiatives to establish a new source of researchers and 
graduates and development of knowledge and technological transfer as instruments of intelligent specialisations at Széchenyi István University”.

\section{References}

[1] In a Tesla Model S, there is no IGBT packing trick

URL https://www.pntpower.com/on-tesla-electric-vehicles-semiconductorpackaging/

[2] E. Barbarini, STMicroelectronics SiC Module Telsa Model 3 Inverter, Power Semiconductor report. 2018.

URL https://www.systemplus.fr/wp-content/uploads/2018/06/SP18413STM_SiC_Module_Tesla_Model_3_Inverter_sample-3.pdf

[3] SiC Power Devices and Modules, ROHM Semiconductor. Application note. 2013.

URL https://www.rohm.com/documents/11308/2420552/SiC_power_devicecatalog.pdf

[4] A. S. Abdelrahman, Z. Erdem, Y. Attia, M. Z. Youssef, Wide Bandgap Devices in Electric Vehicle Converters: A Performance Survey, Canadian Journal of Electrical and Computer Engineering, 41 (1) (2018) pp. 45-54. doi: 10.1109/CJECE.2018.2807780

[5] L. Stephen, A. Robert, Fundamentals of Gallium Nitride Power Transistors. Application note. 2011.

URL https://epc-co.com/epc/Portals/0/epc/documents/producttraining/Appnote_GaNfundamentals.pdf

[6] Design with GaN Enhancement mode HEMT. GN001 Application note. 2018.

URL https://gansystems.com/wpcontent/uploads/2018/02/GN001_Design_with_GaN_EHEMT_180228-1.pdf

[7] Z. John Shen, Power Semiconductor Devices for Hybrid, Electric, and Fuel Cell Vehicles, Proceedings of the IEEE, 95(4) (2017) pp. $778-789$. doi: 10.1109/JPROC.2006.890118

[8] M.H. Rashid, Power Electronics Handbook, $3^{\text {rd }}$ Edition, ButterworthHeinemann, 2007. 
[9] H. Kakitani, R. Takeda, Selecting the Best Power Device for Power Electronics Circuit Design through Gate Charge Characterisation, Elektronika, 56 (10) (2015) pp. 55-59.

[10] Z. Puklus, Power electronics, Universitas-Győr Nonprofit Kft, 2007, in Hungarian

[11] Paralleling of IGBTs, ON Semiconductor. Application note. 2014. URL https://www.onsemi.com/pub/Collateral/AND9100-D.PDF

[12] A. Bhalla, Practical considerations when comparing $\mathrm{SiC}$ and $\mathrm{GaN}$ in power applications. Application note. 2018.

URL http://unitedsic.com/wp-content/uploads/2018/07/Practicalconsiderations-when-comparing-SiC-and-GaN-in-power-applications.pdf 